\title{
THE FOURIER COEFFICIENTS OF EISENSTEIN SERIES NEWFORMS
}

\author{
BENJAMIN LINOWITZ AND LOLA THOMPSON
}

\begin{abstract}
In this article, we study the Fourier coefficients of Eisenstein series newforms. We obtain a sharp refinement of the strong multiplicity-one theorem by showing that the density of primes $p$ for which the $p^{\text {th }}$ Hecke eigenvalues of two distinct Eisenstein series newforms differ is of the form $1 / n$ for some $n \geq 2$. Additionally, we show that if $f$ is an Eisenstein series newform whose Fourier coefficients $a_{f}(n)$ are real then there is a constant $\delta>0$ such that the sequence $\left(a_{f}(n)\right)_{n \leq x}$ has at least $\delta x$ sign changes.
\end{abstract}

\section{INTRODUCTION}

An important problem in the theory of modular forms is to determine the extent to which a newform is determined by its Fourier coefficients. A similar problem asks for characterizations of a newform that enable one to distinguish it from other newforms. The classical strong multiplicity-one theorem, for instance, shows that if the $p^{\text {th }}$ Fourier coefficients of two (normalized) cuspidal newforms agree for all but finitely many primes $p$ then the two newforms are equal. Statistical refinements of results such as this are of great interest. With respect to the strong multiplicity-one theorem, Ramakrishnan [9] has shown that, if the $p^{\text {th }}$ Fourier coefficients of two cuspidal newforms agree for any set of primes having density greater than $7 / 8$, then the two newforms are equal. See Walji [10] for a further refinement.

A related problem that has attracted a great deal of attention in recent years is to consider a newform whose Hecke eigenvalues are real and determine the extent to which the newform is determined by the signs of its Hecke eigenvalues (see $[3,4,6,7]$ ). Some of the many questions that have been addressed in this context are bounds on the first sign change in the sequence of Hecke eigenvalues (a variant of the classical least quadratic non-residue problem in analytic number theory), determining how often the sequence of signs of Fourier coefficients of a newform agree with a fixed sequence of signs, and counting the number of sign changes in the sequence of Fourier coefficients up to $x$.

In a previous paper of the authors [5], the aforementioned problems were considered for the Fourier coefficients of Eisenstein series newforms. It was shown in [5, Theorem 5.4], for instance, that an Eisenstein series newform is determined by the signs of its $p^{\text {th }}$ Fourier coefficients for any set of primes having density greater than $1 / 2$. (Note that by using quadratic twists the authors were able to produce examples which show that this bound is best possible.) In this paper we continue our study of the Fourier coefficients of Eisenstein series newforms. With respect to the strong multiplicity-one theorem, we prove a new result (Theorem 3.4) that implies:

Theorem 1. Let $f, g$ be Eisenstein series newforms of weight $k$ and levels $N, M$. Then the density of the set of primes $p$ for which the $p^{\text {th }}$ Fourier coefficients of $f$ and $g$ agree is of the form $1 / d$ for some divisor $d$ of $\varphi(\operatorname{lcm}[N, M])$, where $\varphi$ is the Euler totient function. Moreover, as $g$ varies over all Eisenstein series newforms of weight $k$, every fraction of the 
form $1 / n$ occurs as the density of primes for which the $p^{\text {th }}$ Fourier coefficients of $f$ and $g$ agree.

Let $f$ be a cuspidal newform for the full modular group with Hecke eigenvalues $\lambda_{f}(n)$. Matomäki and Radziwiłł have recently proven [7] that there is a positive constant $\delta$ such that, for $x$ sufficiently large, the sequence $\left(\lambda_{f}(n)\right)_{n \leq x}$ has at least $\delta x$ sign changes. In Theorem 4.6 we prove an analogous result for the Fourier coefficients of Eisenstein series newforms with real coefficients. Our result can be reformulated in the following manner:

Theorem 2. Let $f$ be an Eisenstein series newform whose Fourier coefficients $a_{f}(n)$ are real numbers. There exists a positive constant $\delta$ such that, for $x$ sufficiently large, the sequence $\left(a_{f}(n)\right)_{n \leq x}$ has at least $\delta x$ sign changes. Moreover, half of the non-zero $a_{f}(n)$ are positive and the other half are negative.

We now discuss the organization of the paper. In Section 2 we review the newform theory of Eisenstein series. This theory was developed by Weisinger [12] in his (unpublished) thesis and extended to Hilbert modular forms by the first author and Atwill [1]. In Section 3 we prove a refinement of the strong multiplicity-one theorem for Eisenstein series newforms. In Section 4 we prove a result concerning the number of sign changes of the Fourier coefficients of an Eisenstein series newform.

\section{A NEWFORM THEORY FOR EISENSTEIN SERIES}

Given a positive integer $N$, a Dirichlet character $\chi$ modulo $N$ and an integer $k \geq 3$, we let $M_{k}(N, \chi)$ be the complex vector space of modular forms for $\Gamma_{0}(N)$ with weight $k$ and character $\chi$. Let $E_{k}(N, \chi)$ denote the subspace of Eisenstein series and $S_{k}(N, \chi)$ denote the subspace of cusp forms. For a prime $p$, we will denote by $T_{p}$ the $p^{\text {th }}$ Hecke operator and recall that $T_{p}$ preserves both $E_{k}(N, \chi)$ and $S_{k}(N, \chi)$. Additionally, for a positive integer $d$, we denote by $B_{d}$ the shift operator defined by

$$
f\left|B_{d}=d^{-k / 2} f\right|\left(\begin{array}{ll}
d & 0 \\
0 & 1
\end{array}\right)
$$

for $f \in M_{k}(N, \chi)$. In terms of Fourier coefficients, if

$$
f=\sum_{n=0}^{\infty} a_{f}(n) q^{n},
$$

where $q=e^{2 \pi i z}$, then

$$
f \mid B_{d}=\sum_{n=0}^{\infty} a_{f}(n) q^{n d} .
$$

This operator maps $M_{k}(N, \chi)$ to $M_{k}(d N, \chi)$ and takes Eisenstein series to Eisenstein series and cusp forms to cusp forms. We note that if $(d, p)=1$ then $B_{d} T_{p}=T_{p} B_{d}$.

Let $N_{1}, N_{2}$ be positive integers such that $N=N_{1} N_{2}$ and let $\chi_{1}, \chi_{2}$ be Dirichlet characters modulo $N_{1}, N_{2}$ for which $\left(\chi_{1} \chi_{2}\right)(-1)=(-1)^{k}$. We now define a modified sum-of-divisors function by

$$
\sigma_{\chi_{1}, \chi_{2}}^{k-1}(n)=\sum_{d \mid n} \chi_{1}(n / d) \chi_{2}(d) d^{k-1}
$$


Associated to the triple $\left(\chi_{1}, \chi_{2}, k\right)$ is a function

$$
E\left(\chi_{1}, \chi_{2}, k\right)=\frac{\delta\left(\chi_{1}\right)}{2} L\left(1-k, \chi_{2}\right)+\sum_{n \geq 1} \sigma_{\chi_{1}, \chi_{2}}^{k-1}(n) q^{n},
$$

where $q=e^{2 \pi i z}, L\left(s, \chi_{2}\right)$ is the Dirichlet $L$-function associated to $\chi_{2}$, and $\delta\left(\chi_{1}\right)=1$ if $\chi_{1}$ is principal and equal to 0 otherwise. It is well-known that $E\left(\chi_{1}, \chi_{2}, k\right) \in E_{k}(N, \chi)$ for $\chi=\chi_{1} \chi_{2}($ see $[2$, Chapter 5]).

Definition. We will say that the Eisenstein series $E\left(\chi_{1}, \chi_{2}, k\right)$ is a newform if the characters $\chi_{1}, \chi_{2}$ are primitive.

The following proposition shows that, just like the cuspidal setting, Eisenstein series newforms are eigenforms for all of the Hecke operators $T_{p}$ and have their $T_{p}^{t h}$-eigenvalue equal to their $p^{\text {th }}$ Fourier coefficient.

Proposition 2.1. Let $E\left(\chi_{1}, \chi_{2}, k\right)$ be as above. Then for every prime $p$ we have

$$
E\left(\chi_{1}, \chi_{2}, k\right) \mid T_{p}=\sigma_{\chi_{1}, \chi_{2}}^{k-1}(p) \cdot E\left(\chi_{1}, \chi_{2}, k\right) .
$$

Proof. If $p \nmid N$ then the desired equality is easily verified by examining the action of $T_{p}$ on the Fourier coefficients of $E\left(\chi_{1}, \chi_{2}, k\right)$. When $p \mid N$ the equality follows from Proposition 3.15 of $[1]$.

Denote by $E_{k}^{\text {new }}(N, \chi)$ the subspace of $E_{k}(N, \chi)$ spanned by newforms of exact level $N$ (i.e., newforms of level $N$ which are not shifts of newforms of level $M<N$ ). The following result (Proposition 3.11 of [1]) shows that, as in the setting of cusp forms, every Eisenstein series can be expressed uniquely as a linear combination of shifts of newforms.

Theorem 2.2. Let the notation be as above. We have the following decompsiton of $E_{k}(N, \chi)$ :

$$
E_{k}(N, \chi)=\bigoplus_{\operatorname{cond}(\chi)|M| N} \bigoplus_{d \mid N M^{-1}} E_{k}^{n e w}(M, \chi) \mid B_{d} .
$$

\section{The Strong MUltiplicity-One TheOREM AND MATCHING DENSITIES}

In this section we will recall a refinement of the strong multiplicity-one theorem proven by the authors in [5, Section 5]. This refinement shows that if the set of primes at which the $p^{\text {th }}$ Hecke eigenvalues of two Eisenstein series newforms coincide has a sufficiently large Dirichlet density then the two newforms are equal. This result raises a number of questions which we will address in this section. For instance, if $f$ and $g$ are two Eisenstein series newforms, what can one say about the density of the set of primes for which their Hecke eigenvalues coincide? In order to address these questions we introduce some useful terminology developed by Walji [11].

Definition. Let $f, g$ be Eisenstein series newforms. The matching density of $f$ and $g$ is the Dirichlet density, if it exists, of the set of primes $p$ for which

$$
a_{f}(p)=a_{g}(p) .
$$

We will denote this matching density by $\delta(f, g)$.

The first strong multiplicity-one theorem in the setting of Eisenstein series was proven by Weisinger [12], who showed that if $f$ and $g$ are Eisenstein series newforms for which $a_{f}(p)=a_{g}(p)$ for all but finitely many primes $p$ then $f=g$. One of the main results of [5, Theorem 5.1] is the following refinement of Weisinger's result. 
Theorem 3.1. Let $f \in E_{k}\left(N, \chi_{f}\right)$ and $g \in E_{k^{\prime}}\left(M, \chi_{g}\right)$ be normalized newforms such that $\delta(f, g)>1 / 2$. Then $k=k^{\prime}, N=M, \chi_{f}=\chi_{g}$ and $f=g$.

We note that Theorem 3.1 is best possible in that one may easily obtain, using quadratic twists, pairs of newforms whose Hecke eigenvalues agree for a set of primes having density $1 / 2$. Indeed, suppose that $f \in E_{k}\left(N, \chi_{f}\right)$ is a newform and that $\theta$ is a quadratic Dirichlet character whose conductor is coprime to $N$. Then by [1, Corollary 5.5], the character twist $f \otimes \theta$ is a newform. Moreover, the $p^{t h}$ coefficient of $f \otimes \theta$ is equal to $a_{f}(p)$ if $\theta(p)=1$ and is equal to $-a_{f}(p)$ if $\theta(p)=-1$. As each of these cases occurs for a set of primes having density $1 / 2$ and the set of primes for which $a_{f}(p)=0$ is finite, we have $\delta(f, f \otimes \theta)=1 / 2$.

Note that the authors have also shown [5, Theorem 5.3] that, in essence, the above quadratic twist construction is the only way of producing newforms $f, g$ for which $\delta(f, g)=1 / 2$. This raises the interesting question of what the maximal value of $\delta(f, g)$ is for non-twistequivalent $f$ and $g$. In what follows, we will prove a theorem (Theorem 3.4) which has as an immediate consequence that the maximal such value is $1 / 3$. The following example of non-twist-equivalent Eisenstein newforms with $\delta(f, g)=1 / 3$ shows that this maximal value is indeed attained.

Example 3.2. Let $\chi$ denote the Dirichlet character modulo 7 defined by $\chi(3)=\zeta_{6}$ and let $\psi$ be the Dirichlet character modulo 7 defined by $\psi(3)=-1$. Then $\chi$ and $\psi$ are primitive characters of conductor 7 with the property that, for a prime $p \neq 7$, one has $\chi(p)=\psi(p)$ if and only if $p \equiv \pm 1(\bmod 7)$. In particular the density of primes $p$ for which $\chi(p)=\psi(p)$ is exactly $1 / 3$. Now let $\theta$ be the trivial character (primitive of conductor 1 ) and define $f=E(\theta, \chi, k)$ and $g=E(\theta, \psi, k)$ for any odd integer $k \geq 3$. Then $f$ and $g$ are Eisenstein series newforms of level 7 which are clearly not twist-equivalent and satisfy $\delta(f, g)=1 / 3$.

In order to prove Theorem 3.4 we will employ the following lemma (for a proof, see $[5$, Lemma 5.2]).

Lemma 3.3. Let $\chi_{1}, \chi_{2}, \psi_{1}, \psi_{2}$ be Dirichlet characters and let $k \geq 2$ be an integer. There exists a positive constant $p_{0}$ such that for all primes $p>p_{0}$, if

$$
\chi_{1}(p)+\chi_{2}(p) p^{k-1}=\psi_{1}(p)+\psi_{2}(p) p^{k-1}
$$

then $\chi_{1}(p)=\psi_{1}(p)$ and $\chi_{2}(p)=\psi_{2}(p)$.

Theorem 3.4. Let $f \in E_{k}\left(N, \chi_{f}\right)$ and $g \in E_{k^{\prime}}\left(M, \chi_{g}\right)$ be newforms.

(1) $\delta(f, g)=0$ if and only if $k \neq k^{\prime}$.

(2) If $k=k^{\prime}$ then $\delta(f, g)=1 / d$ for some divisor $d$ of $\varphi(\operatorname{lcm}[N, M])$.

(3) For every positive integer $n$, there exists a newform $g$ such that $\delta(f, g)=1 / n$.

Proof. Write $f=E\left(\chi_{1}, \chi_{2}, k\right)$ and $g=E\left(\psi_{1}, \psi_{2}, k^{\prime}\right)$ and note that $a_{f}(p)=\chi_{1}(p)+\chi_{2}(p) p^{k-1}$ and $a_{g}(p)=\psi_{1}(p)+\psi_{2}(p) p^{k^{\prime}-1}$. Fix $\epsilon \in(0,1)$. For all sufficiently large primes $p$, we have $\left|a_{f}(p)\right| \in\left(p^{k-1-\epsilon}, p^{k-1+\epsilon}\right)$ and $\left|a_{g}(p)\right| \in\left(p^{k^{\prime}-1-\epsilon}, p^{k^{\prime}-1+\epsilon}\right)$. It follows that $k \neq k^{\prime}$ implies $\delta(f, g)=0$, as these two intervals intersect if and only if $k=k^{\prime}$. To prove the converse, we must show that if $k=k^{\prime}$ then $\delta(f, g)>0$. Suppose therefore that $k=k^{\prime}$. If $p \equiv 1$ $(\bmod \operatorname{lcm}[N, M])$ then

$$
a_{f}(p)=\chi_{1}(p)+\chi_{2}(p) p^{k-1}=1+p^{k-1}=\psi_{1}(p)+\psi_{2}(p) p^{k-1}=a_{g}(p) .
$$

Assertion (1) now follows from the fact that the set of primes congruent to 1 modulo $\operatorname{lcm}[N, M]$ has positive density. 
Henceforth we will assume that $k=k^{\prime}$. We now prove (2). Denote by $\chi_{1}^{\prime}, \chi_{2}^{\prime}, \psi_{1}^{\prime}, \psi_{2}^{\prime}$ the characters modulo $\operatorname{lcm}[N, M]$ induced by $\chi_{1}, \chi_{2}, \psi_{1}, \psi_{2}$. Let $p_{1}, \ldots, p_{\varphi(\operatorname{lcm}[N, M])}$ be primes representing the residue classes of $(\mathbf{Z} / \operatorname{lcm}[N, M] \mathbf{Z})^{\times}$which are all large enough that Lemma 3.3 holds. In particular, if $a_{f}\left(p_{i}\right)=a_{g}\left(p_{i}\right)$ then Lemma 3.3 implies that $\chi_{1}^{\prime}\left(p_{i}\right)=\psi_{1}^{\prime}\left(p_{i}\right)$ and $\chi_{2}^{\prime}\left(p_{i}\right)=\psi_{2}^{\prime}\left(p_{i}\right)$, hence $\chi_{1}\left(p_{i}\right)=\psi_{1}\left(p_{i}\right)$ and $\chi_{2}\left(p_{i}\right)=\psi_{2}\left(p_{i}\right)$. Conversely, if $\chi_{1}\left(p_{i}\right)=\psi_{1}\left(p_{i}\right)$ and $\chi_{2}\left(p_{i}\right)=\psi_{2}\left(p_{i}\right)$ then we will have $a_{f}(p)=a_{g}(p)$ for every prime $p \equiv p_{i}(\bmod \operatorname{lcm}[N, M])$. From this we conclude that if there are precisely $s$ values of $i$ for which $\chi_{1}\left(p_{i}\right)=\psi_{1}\left(p_{i}\right)$ and $\chi_{2}\left(p_{i}\right)=\psi_{2}\left(p_{i}\right)$, then $\delta(f, g)=s / \varphi(\operatorname{lcm}[N, M])$. In particular, to prove assertion $(2)$, it suffices to show that $s$ is a divisor of $\varphi(\operatorname{lcm}[N, M])$. But this is clear, as the set of such primes $p_{i}$ comprise a subgroup of $(\mathbf{Z} / \operatorname{lcm}[N, M] \mathbf{Z})^{\times}$. Explicitly, this subgroup is $\operatorname{ker}\left(\chi_{1}^{\prime} \overline{\psi_{1}^{\prime}}\right) \cap$

$\operatorname{ker}\left(\chi_{2}^{\prime} \overline{\psi_{2}^{\prime}}\right)$, where $\chi_{1}^{\prime} \overline{\psi_{1}^{\prime}}$ and $\chi_{2}^{\prime} \overline{\psi_{2}^{\prime}}$ are regarded as homomorphisms from $(\mathbf{Z} / \operatorname{lcm}[N, M] \mathbf{Z})^{\times}$to $\mathbf{C}^{\times}$.

We now prove (3). Fix a positive integer $n$. We will construct a newform $g$ for which $\delta(f, g)=1 / n$. As we trivially have $\delta(f, f)=1$, we may assume that $n \geq 2$. Let $q$ be a prime which does not divide $N$ and which satisfies $q \equiv 1(\bmod n)$. As $(\mathbf{Z} / q \mathbf{Z})^{\times}$is cyclic of order $q-1$, there exists a Dirichlet character $\theta_{0}$ modulo $q$ which has order $q-1$. Let $\theta=\theta_{0}^{(q-1) / n}$ and note that the density of primes $p$ for which $\theta(p)=1$ is $1 / n$. By [1, Corollary 5.5], $f \otimes \theta$ is a newform, and by definition, $a_{g \otimes \theta}(p)=\theta(p) a_{f}(p)$. It follows that $\delta(f, f \otimes \theta)=1 / n$, proving assertion (3).

Remark. We note that the third assertion of Theorem 3.4 can alternatively be proven by associating to every Eisenstein series newform a reducible two-dimensional Galois representation (which would arise from the pair of Dirichlet characters defining the newform) and then using the fact that the matching densities of abelian representations are of the form $\{1 / n: n \geq 2\}$ (see [11]).

\section{Sign Changes of Fourier coefficients}

A series of recent papers studies when the first negative Fourier coefficient occurs. In the cusp form case, we have a well-known theorem of Kowalski, Lau, Soundararajan and Wu [4], which was recently improved by Matomäki [6]. In order to state these results, we denote by $f$ a cuspidal newform of weight $k$, level $N$, and trivial nebentypus. Note that the restriction to trivial nebentypus ensures that all of the Fourier coefficients of $f$ are real. We denote by $\lambda_{f}(n)$ the normalized Fourier coefficients of $f$.

Theorem 4.1 (Kowalski, Lau, Soundararajan, Wu, 2010). For $k$ even and $(n, N)=1$, we have $\lambda_{f}(n)<0$ for some $n \ll\left(k^{2} N\right)^{9 / 20}$.

Theorem 4.2 (Matomäki, 2012). For $k$ even and $(n, N)=1$, we have $\lambda_{f}(n)<0$ for some $n \ll\left(k^{2} N\right)^{3 / 8}$.

Matomäki and Radziwiłł [7] went on to prove a stronger result in the special case where $N=1$.

Theorem 4.3 (Matomäki and Radziwilł, 2015). For $N=1$, there exists a positive constant $\delta$ such that, for $x$ sufficiently large, the sequence $\left(\lambda_{f}(n)\right)_{n \leq x}$ has at least $\delta x$ sign changes. Furthermore, half of the non-zero $\lambda_{f}(n)$ are positive and half are negative. 
We can obtain analogous results for Eisenstein series newforms. Recall that if $f$ is an Eisenstein series newform then $f$ is associated to a triple $\left(\chi_{1}, \chi_{2}, k\right)$ and satisfies

$$
f=E\left(\chi_{1}, \chi_{2}, k\right)=\frac{\delta\left(\chi_{1}\right)}{2} L\left(1-k, \chi_{2}\right)+\sum_{n \geq 1} \sigma_{\chi_{1}, \chi_{2}}^{k-1}(n) q^{n},
$$

where $q=e^{2 \pi i z}, L\left(s, \chi_{2}\right)$ is the Dirichlet $L$-function associated to $\chi_{2}$ and $\delta\left(\chi_{1}\right)=1$ if $\chi_{1}$ is principal and equal to 0 otherwise. In order to ensure that our Fourier coefficients are real, we will only consider Eisenstein series newforms associated to quadratic Dirichlet characters. We additionally assume that $k \geq 3$.

Our proofs all rely on a single key lemma, which shows that understanding the signs of Fourier coefficients of Eisenstein series newforms amounts to understanding the behavior of certain quadratic Dirichlet characters. The proof of the key lemma first appeared in [5]. We reproduce it here for the sake of completeness.

Lemma 4.4. If $(n, N)=1$ and $\chi_{1}, \chi_{2}$ are quadratic, the sign of $\sigma_{\chi_{1}, \chi_{2}}^{k-1}(n)$ is completely determined by the behavior of $\chi_{2}(n)$.

Proof. Starting with the definition of $\sigma_{\chi_{1}, \chi_{2}}^{k-1}(n)$ given in (2.1), we can separate off the $d=n$ term, which gives us

$$
\begin{aligned}
\sigma_{\chi_{1}, \chi_{2}}^{k-1}(n) & =\sum_{d \mid n} \chi_{1}(n / d) \chi_{2}(d) d^{k-1} \\
& =\chi_{2}(n) n^{k-1}+\sum_{\substack{d \mid n \\
d<n}} \chi_{1}(n / d) \chi_{2}(d) d^{k-1} \\
& =n^{k-1}\left(\chi_{2}(n)+\sum_{\substack{d \mid n \\
d<n}} \frac{\chi_{1}(n / d) \chi_{2}(d)}{(n / d)^{k-1}}\right) .
\end{aligned}
$$

Consider the summation over the proper divisors of $n$ in (4.1). Since $\left|\chi_{1}(d)\right|,\left|\chi_{2}(d)\right| \leq 1$ for all $d \in \mathbf{Z}^{+}$, we can bound each numerator from above by 1 , which yields

$$
\sum_{\substack{d \mid n \\ d<n}} \frac{\chi_{1}(n / d) \chi_{2}(d)}{(n / d)^{k-1}} \leq \sum_{\substack{d \mid n \\ d<n}} \frac{1}{(n / d)^{k-1}} \leq \sum_{m \geq 2} \frac{1}{m^{k-1}}=\zeta(k-1)-1<1 .
$$

Thus, the $\chi_{2}(n) n^{k-1}$ term dominates in (4.1) and so we have

$$
\operatorname{sgn} \sigma_{\chi_{1}, \chi_{2}}^{k-1}(n)=\chi_{2}(n) \text {. }
$$

In [5], we used Lemma 4.4 to demonstrate when the first sign change occurs.

Theorem 4.5. Let $p_{0}$ represent the smallest prime for which $\sigma_{\chi_{1}, \chi_{2}}^{k-1}\left(p_{0}\right)<0$. Then, for any fixed $\varepsilon>0$, we have

$$
p_{0} \ll_{\varepsilon} N^{\frac{1}{4 \sqrt{e}}+\varepsilon} .
$$

Note that the $1 / 4 \sqrt{e}$ in the exponent comes from the classical Burgess bound for the least quadratic non-residue. We can use Theorem 4.5 to obtain an analogue of Theorem 4.3 for Eisenstein series: 
Theorem 4.6. Let $f$ be an Eisenstein series newform with Fourier coefficients $\sigma_{\chi_{1}, \chi_{2}}^{k-1}(n)$. There exists a positive constant $\delta$ such that, for $x$ sufficiently large, the sequence $\left(\sigma_{\chi_{1}, \chi_{2}}^{k-1}(n)\right)_{n \leq x}$ has at least $\delta x$ sign changes. Moreover, half of the non-zero $\sigma_{\chi_{1}, \chi_{2}}^{k-1}(n)$ are positive and the other half are negative.

The proof of the first part of this theorem relies on a recent result of Matomäki and Radziwiłł that appeared in [8].

Theorem 4.7 (Matomäki \& Radziwiłt, 2016). Let $f: \mathbf{N} \rightarrow \mathbf{R}$ be a multiplicative function. Then $f(n)$ has a positive proportion of sign changes if and only if $f(n)<0$ for some integer $n>0$ and $f(n) \neq 0$ for a positive proportion of integers $n$.

Proof of Theorem 4.6. For the first assertion, recall that we are assuming that $\chi_{2}$ is a quadratic character $\left(\bmod N_{2}\right)$. From Lemma 4.4, the sign of $\sigma_{\chi_{1}, \chi_{2}}^{k-1}$ is completely determined by the sign of $\chi_{2}$. In particular, we can rewrite

$$
\operatorname{sgn} \sigma_{\chi_{1}, \chi_{2}}^{k-1}(n)=\prod_{p^{\ell} \| n}\left(\chi_{2}(p)\right)^{\ell} .
$$

Call this function $g(n)$. The function $g$ is multiplicative because it is a product of $\chi_{2}(p)$ 's and $\chi_{2}$ is totally multiplicative. By Theorem 4.5, we know that $g(n)<0$ for some integer $n>0$. We now show that $g(n) \neq 0$ for a positive proportion of integers $n$. Indeed, if $n \equiv 1\left(\bmod N_{2}\right)$ then $\chi_{2}(n)=1$ since the conductor of $\chi_{2}$ is $N_{2}$. It follows that, for $n \equiv 1$ $\left(\bmod N_{2}\right)$, we have $\operatorname{sgn}\left(\chi_{2}(n)\right)=1$. Hence, by Lemma 4.4, $\operatorname{sgn}\left(\sigma_{\chi_{1}, \chi_{2}}^{k-1}\right)=1$, so $g(n)=1$. Because the density of integers $n$ such that $n \equiv 1\left(\bmod N_{2}\right)$ is positive, we see that $g(n) \neq 0$ for a positive proportion of integers. Therefore the hypotheses for Theorem 4.7 are met and we have that $g$ changes sign a positive proportion of the time. In other words, there exists a positive constant $\delta$ such that the sequence $\left(\sigma_{\chi_{1}, \chi_{2}}^{k-1}(n)\right)_{n \leq x}$ has at least $\delta x$ sign changes.

For the second assertion, we use the fact that the Dirichlet character $\chi_{2}(n)=-1$ half of the time and $\chi_{2}(n)=1$ the other half of the time. By Lemma 4.4, it follows that half of the non-zero $\sigma_{\chi_{1}, \chi_{2}}^{k-1}(n)$ are positive and the other half are negative. (Note: The proof of this second claim originally appeared as Corollary 3.2 in [5].)

\section{ACKNOWLEDGEMENTS}

The authors would like to thank the organizers of the $3^{r d}$ Building Bridges Workshop on Automorphic Forms and Related Topics for providing them with the opportunity to participate in a wonderful conference. The second author is supported by an AMS Simons Travel Grant and by a fellowship from the Max Planck Institute for Mathematics.

\section{REFERENCES}

1. Timothy W. Atwill and Benjamin Linowitz, Newform theory for Hilbert Eisenstein series, Ramanujan J. 30 (2013), no. 2, 257-278.

2. Fred Diamond and Jerry Shurman, A first course in modular forms, Graduate Texts in Mathematics, vol. 228, Springer-Verlag, New York, 2005. MR 2112196

3. H. Iwaniec, W. Kohnen, and J. Sengupta, The first negative Hecke eigenvalue, Int. J. Number Theory 3 (2007), no. 3, 355-363. MR 2352824

4. E. Kowalski, Y.-K. Lau, K. Soundararajan, and J. Wu, On modular signs, Math. Proc. Cambridge Philos. Soc. 149 (2010), no. 3, 389-411.

5. B. Linowitz and L. Thompson, The sign changes of Fourier coefficients of Eisenstein series, Ramanujan J. 37 (2015), no. 2, 223-241. 
6. K. Matomäki, On signs of Fourier coefficients of cusp forms, Math. Proc. Cambridge Philos. Soc. 152 (2012), no. 2, 207-222.

7. K. Matomäki and M. Radziwiłł, Sign changes of Hecke eigenvalues, Geom. Funct. Anal. 25 (2015), no. 6, 1937-1955.

8. __ Multiplicative functions in short intervals, Ann. Math. 183 (2016), 1015-1056.

9. D. Ramakrishnan, A refinement of the strong multiplicity one theorem for $\mathrm{GL}(2)$. appendix to: l-adic representations associated to modular forms over imaginary quadratic fields. ii, Invent. Math. 116 (1994), no. 1-3, 619-643.

10. Nahid Walji, Further refinement of strong multiplicity one for GL(2), Trans. Amer. Math. Soc. 366 (2014), no. 9, 4987-5007. MR 3217707

11. __ Matching densities for Galois representations, Proc. Amer. Math. Soc. 144 (2016), no. 8, 33093316. MR 3503699

12. J. Weisinger, Some results on classical Eisenstein series and modular forms over function fields, Thesis, Harvard Univ. (1977).

Department of Mathematics, Oberlin College, Oberlin, OH 44074

E-mail address: benjamin.linowitz@oberlin.edu

Department of Mathematics, Oberlin College, Oberlin, OH 44074

Max Planck Institute for Mathematics, Bonn, Germany 53111

E-mail address: lola.thompson@oberlin.edu 